\title{
Use of Outpatient Parenteral Antimicrobial Therapy (OPAT) in Chronic Osteomyelitis
}

\author{
Marcano-Lozada $\mathbf{M}^{1,2 *}$ \\ 1Medical Microbiology Specialist, Angios Vascular Center \& Wound Clinic, Venezuela \\ 2Aggregate Professor Microbiology Department, "J.M. Vargas" School of Medicine, \\ Universidad Central de Venezuela, Venezuela
}

Editorial

Volume 3 Issue 1

Received Date: January 22, 2019

Published Date: February 01, 2019

*Corresponding author: Marcel Marcano-Lozada, Aggregate Professor Microbiology Department, "J.M. Vargas" School of Medicine, Universidad Central de Venezuela, Caracas, Venezuela, E-mail: marcelmarcano@gmail.com

\section{Editorial}

The main goal in today's therapy for acute or chronic infections is to achieve the cure in the minimum time with the minimum (and more effective) treatment, and this concept implicate in many cases the use of parenteral antimicrobial therapy, with the concomitant hospitalization to receive this modality of treatment (especially intravenous medication). But the increase in costs, the need of the hospital beds for very sick patients, the risk to acquire a nosocomial infection due to a superbug, are major factors to consider alternatives to continue with the administration of the antimicrobials in parenteral modality in the community setting (especially at the intravenous -IV-access), to preserve the power of the antibacterial treatment outside the hospital walls.

Additional factors come with the use of parenteral antimicrobial therapy in patients' home or outside the hospitalization setting, known as OPAT (Outpatient Parenteral Antimicrobial Therapy) such as: quickly recovery \& life quality improvement, possibility to an early return to work, that keeps the "routine perception" of normal lifestyle for the patient, resulting in major adherence to the treatment, because it doesn't interfere with the patient daily activities. The concept of outpatient parenteral antimicrobial therapy for the management of some infections is a great alternative with a lot of benefits for the healthcare providers, insurance companies, doctors \& patients. As a personal experience, I begin using OPAT for the treatment of chronic osteomyelitis, because this complex pathology requires an initial stage of strong IV drugs (known as "attack phase") to achieve the bactericidal concentration in the bone, and the duration of this treatment is among 3 to 6 weeks, also is necessary the combination with drugs with anti-biofilm spectrum like rifampicin for example, and then this antimicrobial treatment can be switch to oral drugs in the "consolidation phase" for 3 to 6 weeks or more depending of the combination. Initially, drugs should be administered in a special healthcare setting known as "outpatient infusion center", a facility with comfortable access and furniture, quiet \& relaxing (we offer books, tv, music to make a more pleasant time), a concept taken from the oncological infusion units \& biological therapy infusion units; then, the experience training our nurses and paramedical personnel bring us the opportunity to administered OPAT in the patient's home, and it was particularly useful in cases when the patient has reduced mobility (wheelchairs, elderly people, inmates, etc.), and increases the adherence to the therapy [1].

Antimicrobial drugs for OPAT needs to have some special characteristics like stability (nor degradation after reconstitution or preparation for infusion), easy infusion or injection (bolus is the preferred administration), convenient posology regimen (one or two daily dose), short infusion time (no more than an hour), low adverseevents related with administration (infections, phlebitis, allergic reactions, etc.), low (or not) interactions with other concomitant medications, a favorable pharmacoeconomic profile (administration in the outpatient setting should be decreased healthcare associated costs) and flexibility to be administered at outpatient setting in the hospital (treatment rooms) or in patient's home [2]. 


\section{Journal of Infectious Diseases \& Travel Medicine}

In the particular cases of chronic osteomyelitis, the drugs need to exhibit antibiofilm activity and be able to concentrate in an accumulative way in the bone tissue, and present a bactericidal mechanism of action. The low induction of inflammatory phenomena related to the drug cidality (bactericidal activity) is a very desirable plus. From the resistance point of view, the low induction of antimicrobial resistance is a must, in order to keep the drug use for an extended period of time without losing efficacy [3].

The preferred drug for use in chronic osteomyelitis OPAT is Daptomycin, a cyclic lipopeptide antibiotic drug, approved for complicated skin and soft tissue infections, right-sided infective endocarditis and bacteremia due to Staphylococcus aureus [4], not approved for use in bone infection, but this drug become in the last resource in cases when bone infection due to multi-resistant bacteria (superbugs) is a threat for limb amputation. Its unique mechanism of action that kills Gram-positive bacteria by the disruption of multiple bacterial plasma membrane functions, without penetrating the cytoplasm begins with the insertion of the lipophilic daptomycin tail into the bacterial cell membrane with oligomerization and channel formation causes rapid membrane depolarization and a potassium ion efflux [5]. Arrest of nucleic acids (DNA/RNA) synthesis, toxin production, and protein synthesis follows, resulting in bacterial death without lysis of the cell wall, which gives a further advantage in diseases where inflammatory response associated counter antimicrobial use producing cell lysis (such as meningitis). Daptomycin's synergic effect has been described in vitro with aminoglycosides, oxacillin and other betalactamic, macrolides and rifampicin, is very valuable to biofilm disease infections treatment, and this anti-biofilm activity and reduction of the rifampicin resistance appearance makes a great value. Not antagonism was observed in combination with several antimicrobial agents, only additive, synergistic effect or indifference were reported. Another relevant aspect is daptomycin's per se partial anti-biofilm activity (as monotherapy), and its increases in combinations.

Our initial approaches to other drugs for chronic osteomyelitis OPAT, like vancomycin, doesn't show good results, because the need for use in the hospital facilities, prolonged-time of infusion ( $>60$ minutes, twice a day), high volume of infusion to avoid adverse events related to the infusion (like phlebitis or "red-man syndrome) creates discomfort and risk of hydric overcharge in patients with volume restriction (cardiac heart failure, renal impairment), and the need to adjust dose based on PK/PD parameters, also, the superbugs develop "tolerance" for this antimicrobial, maybe because the necessary cidal level isn't achieved in the bone tissue, so, infections persist although the microorganisms were sensitive to the antibiotic.

We use extremely-high daptomycin doses (major than $10 \mathrm{mg} / \mathrm{Kg} / \mathrm{IV} / \mathrm{OD}$ ), for 4 to 6 weeks, and then was switched to oral agents for consolidation phase for 4 to 6 weeks [6]. The use of daptomycin as monotherapy is controversial, we don't recommend, but in some special and very particular cases, it was the only option against limb amputation [7]. The most effective combination of daptomycin at high dose is with rifampin (standard dose), reserved to biofilm-producer bacteria, for a time of 6 to 8 weeks, follow by a consolidation phase of 4 to 6 weeks of linezolid at standard dose (600 mg BID) plus a macrolide (azithro or clarithromycin) at standard dose, or a onedaily quinolone (moxi or levofloxacin) plus a macrolide $[8,9]$.

Daptomycin's posology is ideal for OPAT use, because one-daily dose adjusted with body weight is a great choice to maintain the most "natural" lifestyle. In cases when renal impairment is present, dose needs to be applied every 48 hours. The potential venous damage in longterm IV treatments is a reality, but we prevent it with the use of central venous catheters (similar to chemotherapy access), with provides a safe, low-infection risk and comfortable way of administration, without the inconvenient rotation of injection sites, catheter-related infection like cellulitis, discomfort (due to phlebitis), etc.

Another important consideration for the OPAT is the time duration for administration, because is not the same using antimicrobial drugs that need a prolonged infusion time, and the advantages of the one daily 30 minutes infusion over other drugs that require more extended periods of administration were remarkable. An alternative to the classic daptomycin infusion is the 2minutes bolus administration [10] that shows great improvements: less volume of infusion (only the reconstituted ones), less equipment needs (no infusion set required), less healthcare personnel time use (only reconstitution time and 2-minutes bolus administration), healthcare costs reduction (less time needed to deliver the treatment, less time of infusion units permanence increase rotation \& availability, etc.), patient's satisfaction (with a very small impact in the daily lifestyle) and increase of adherence (easy to fulfilled regimen).

In resume, my ten years' experience in the management of chronic osteomyelitis was very positive and great using daptomycin's OPAT regimens due to the flexibility and adherence that this treatment modality offers to the patients, with a significant reduction in the 
healthcare associated cost, and the most important, preserving patient's quality of life and improving it (physical, emotional, at work or home). The bacterial control and eradication were reached in the great majority of patients in the attack phase treatment, and it was paired with clinical improvement reported by patients, that ensures that the second phase of treatment (consolidation phase) should be completed to preserve the good results of the first one [11], also, daptomycin's OPAT is the backbone of therapy in cases when antimicrobial drugs availability was limited, especially against biofilm-producer superbugs [12].

\section{References}

1. Chapman AL, Seaton RA, Cooper MA, Hedderwick S, Goodall V, et al. (2012) Good practice recommendations for outpatient parenteral antimicrobial therapy (OPAT) in adults in the UK: a consensus statement. J Antimicrob Chemother 67(5): 1053-1062.

2. Seaton RA, Sharp E, Bezlyak V, Weir CJ (2011) Factors associated with outcome and duration of therapy in outpatient parenteral antibiotic therapy (OPAT) patients with skin and soft-tissue infections. Int J Antimicrob Agents 38(3): 243-248.

3. Seaton RA, Gonzalez-Ramallo VA, Prisco V, MarcanoLozada M, Gonzalez-Ruiz A, et al. (2013) Daptomycin for outpatient parenteral antibiotic therapy: a European registry experience. Int J Antimicrob Agents 41(5): 468-472.

4. (2009) Novartis Europharm Ltd. Cubicin (daptomycin) Summary of Product Characteristics.

5. Tedesco KL, Rybak MJ (2004) Daptomycin. Pharmacotherapy 24(1): 41-57.
6. Marcano-Lozada M, Molero-Leon S (2018) Dawn of the Biofilm Disease: Highlights about Biofilm in Bone and Joint \& Prosthetic Joint Infections Pathogenesis, Diagnosis and Treatment. J Microbiol Modern Tech 3(1): 104.

7. Marcano-Lozada M, Molero-Leon S (2017) BiofilmProducer MRSA Chronic Osteomyelitis in a Diabetic Patient Successfully Treated with Antimicrobial Monotherapy. J Exp Microbiol 4(3): 1-4.

8. Marcano-Lozada M, Molero-Leon S (2016) Highlights of Antimicrobial Use in Osteomyelitis as Prototype of Disease Biofilms in Venezuela. J Exp Microbiol 3(4): 00096.

9. Marcano-Lozada M (2018) Comments of Clinical and Microbiological Experience with Daptomycin in Chronic Osteomyelitis Treatment. Cohesive J Microbiol infect Dis 2(2): 1-3

10. Chakraborty A, Roy S, Loeffler J, Chaves RL (2009) Comparison of the pharmacokinetics, safety and tolerability of daptomycin in healthy adult volunteers following intravenous administration by $30 \mathrm{~min}$ infusion or 2 min injection. J Antimicrob Chemother 64(1): 151-158.

11. Marcano-Lozada M, Molero-Leon S (2018) 7 Years of Experience in Osteomyelitis Management in Caracas, Venezuela. Cohesive J Microbiol infect Dis 2(1): 1-9.

12. Marcano-Lozada M, Molero León, Silvia Elena (2018) Consideraciones diagnósticas y terapéuticas en el manejo de la osteomielitis crónica asociada a microorganismos formadores de biopelículas en Venezuela durante el bienio 2015-2016. VITAE 73: 1. 